\title{
The Challenges of Multicore: Information and Mis-Information
}

\author{
Yale Patt
}

The University of Texas at Austin

\begin{abstract}
Now that we have broken the threshold of one billion transistors on a chip and multi-core has become a reality, a lot of buzz has resulted - from how/why we got here, to what is important, to how we should determine how to effectively use multicore. In this talk, I will examine a number of these new "conventional wisdom" nuggets of information to try to see whether they add value or get in the way. For example: what can we expect multicore to do about saving power consumption? is ILP dead? should sample benchmarks drive future designs? is hardware sequential? should multicore structures be simple? is abstraction a fundamental good? Hopefully, our examinations will help shed some light on where we go from here.
\end{abstract}

